\title{
A CHARACTERIZATION OF ORDER TOPOLOGIES BY MEANS OF MINIMAL $T_{0}$-TOPOLOGIES
}

\section{W. J. THRON AND SUSAN J. ZIMMERMAN}

ABSTRACT. In this article we give a purely topological characterization for a topology $J$ on a set $X$ to be the order topology with respect to some linear order $R$ on $X$, as follows. A topology $J$ on a set $X$ is an order topology iff $(X, J)$ is a $T_{1}$-space and $J$ is the least upper bound of two minimal $T_{0}$-topologies [Theorem 1]. From this we deduce a purely topological description of the usual topology on the set of all real numbers. That is, a topological space $(X, J)$ is homeomorphic to the reals with the usual topology iff $(X, \mathfrak{J})$ is a connected, separable, $T_{1}$-space, and $J$ is the least upper bound of

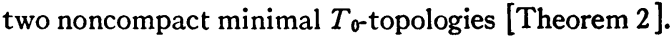

1. Introduction. The concept of the order topology on a linearly ordered set goes back at least to Haar and König (1911) and is the following:

Definition 1. A topology $J$ on a set $X$ is an order topology on $X$ iff there is a linear order $R$ on $X$ such that the sets of the forms

$$
\{y: y<x\} \text { and }\{y: x<y\} \text {, }
$$

where $x \in X$, form a subbase for $J$.

In this definition and in what follows, we use the convention that, if $R$ is a pre-order relation and $\langle a, b\rangle \in R$, we may also write $a \leqq b$. If, in addition, $a \neq b$, we write $a<b$. The symbol $a \geqq b$ means, technically, that $\langle a, b\rangle \in R^{-1}$, where $R^{-1}=\{\langle x, y\rangle:\langle y, x\rangle \in R\}$, and $\left.a\right\rangle b$ is defined similarly. Whenever it is desirable to emphasize the particular relation $R$, we will write $a \leqq_{R} b$ and do likewise for the other three symbols.

There have been, historically, several topological characterizations of intervals in the real line, which are in the same spirit as our Theorem 2. Sierpinski (1917), Hausdorff (1927), and Pauc (1936), among others, gave topological characterizations of the linear continuum $[0,1]$, also known as the Jordan arc. Sierpiński worked within Euclidean $n$-space, while Hausdorff and Pauc used general metric spaces. In 1936 Ward, using a condition similar to Hausdorff's, characterized the open interval $(0,1)$ as a connected locally

Received by the editors March 21, 1970.

AMS 1969 subject classifications. Primary 5456; Secondary 0630.

Key words and phrases. Lattice of topologies, pre-order relation, complete order, dense order, connected space, separable space, nested topology, minimal $T_{0}$-topology. 
connected separable metric space $X$ such that for each $x \in X, X \sim\{x\}$ has precisely two components [15, p. 191]. Franklin and Krishnarao [3] have recently pointed out that Ward's characterization still holds when "metric" is replaced by "regular." Eilenberg in 1941 extended Pauc's result to show that a connected locally connected separable topological space $X$ is homeomorphic to a subinterval of $[0,1]$ iff $\{\langle x, y\rangle: x \neq y\}$ is not connected in the product topology on $X \times X[2$, p. 43].

Both of our theorems depend on the following result, recently obtained by Larson $[7$, p. 453$]$ and Pahk [10, p. 7]. We note that a topology $\Im$ on $X$ is nested iff $\Im$ is a nested family of sets; that is, iff, for all $G, H \in \mathfrak{J}, G \subset H$ or $H \subset G$.

Theorem A. $A T_{0}$-topological space $(X, J)$ is minimal $T_{0}$ iff $\mathrm{J}$ is nested and the set of all complements of point closures, $\{\sim\{\bar{x}\}: x \in X\}$ $\cup\{X\}$, forms a base for $\}$.

2. Proof of Theorem 1. We first give a brief description of the lattice structure on the set of topologies on a set $X$ and then prove two lemmas.

The set, $\Sigma$, of all topologies on a fixed set $X$ forms a lattice when ordered by set inclusion. For any two topologies $J_{1}$ and $J_{2}$ on $X$, the least upper bound, $J_{1} \vee J_{2}$, is the smallest topology containing $J_{1} \cup J_{2}$, and is not, in general, $J_{1} \cup J_{2}$. However, if $B_{1}$ and $B_{2}$ are bases for $J_{1}$ and $J_{2}$, respectively, then $B_{1} \cup B_{2}$ is a subbase for $J_{1} \vee J_{2}$ and $\left\{B_{1} \cap B_{2}: B_{1} \in B_{1}\right.$ and $\left.B_{2} \in B_{2}\right\}$ is a base for $J_{1} \vee J_{2}$.

With each topology $J \in \Sigma$, it is possible to associate a relation $R_{J}$ defined by $\langle a, b\rangle \in R_{\mathfrak{J}}$ iff every open set containing $b$ contains $a$. It is clear that $R_{\Im}$ is always reflexive and transitive and hence a pre-order relation. Ore in his paper of 1943 defined such a relation for each closure operator (not necessarily topological) on $X$. In this more general context, he also proved the first part of Lemma 1 [9, p. 763]. In fact, the function

$$
\mathfrak{I} \stackrel{\varphi}{\rightarrow} R_{\mathfrak{J}}
$$

is an order "antihomomorphism," because $\Im_{1} \subset J_{2} \Rightarrow R_{J_{1}} \supset R_{\Im_{2}}$. Steiner $[13$, p. 383] has shown that, when restricted to the principal topologies on $X, \varphi$ is actually a lattice anti-isomorphism. This same $\varphi$, when restricted to minimal $T_{0}$-topologies on $X$, describes a 1-1 correspondence between the minimal $T_{0}$-topologies on $X$ and the linear order relations on $X$ (see Lemma 2) and provides the basis for the proof of Theorem 1. 
LEMMA 1. If $J$ is any topology on $X$, then

(i) $J$ is $T_{0}$ iff $R_{\mathfrak{J}}$ is a partial order, and

(ii) $\Im$ is nested iff $R_{\mathfrak{J}}$ is such that, for all $a, b \in X,\langle a, b\rangle \in R_{\mathfrak{J}}$ or $\langle b, a\rangle \in R_{\text {J. }}$

Proof. (i) If $J$ is $T_{0}$, then, whenever $\langle x, y\rangle \in R_{\mathfrak{J}}$ and $\langle y, x\rangle \in R_{\mathfrak{J}}, x$ must equal $y$, because every neighborhood of $y$ contains $x$ and every neighborhood of $x$ contains $y$. Thus $R_{\mathfrak{J}}$ is antisymmetric and a partial order.

If $R_{\mathfrak{J}}$ is a partial order, suppose $x$ and $y$ are distinct points of $X$ and that every neighborhood of $x$ contains $y$. That is, $\langle y, x\rangle \in R_{\mathfrak{J}}$ and $x \neq y$. Therefore, since $R_{\mathfrak{J}}$ is a partial order, $\langle x, y\rangle \notin R_{\mathfrak{J}}$ and there is a neighborhood $N_{y}$ of $y$ such that $x \notin N_{y}$.

(ii) If $I$ is nested, let $x, y \in X$ and suppose $\langle x, y\rangle \notin R_{\mathfrak{J}}$. Then there is an open set $G$ with $y \in G$ and $x \notin G$. Let $H$ be any open set containing $x$. Then $x \notin G \Rightarrow H \nsubseteq G \Rightarrow G \subset H \Rightarrow y \in H$. That is, every open set containing $x$ contains $y$ and $\langle y, x\rangle \in R_{\mathfrak{J}}$.

Now let $R_{\mathfrak{J}}$ be such that for all $a, b \in X,\langle a, b\rangle \in R_{\mathfrak{J}}$ or $\langle b, a\rangle \in R_{\mathfrak{J}}$. Let $G, H \in J$ and assume $H \nsubseteq G$. Then there is an $h \in H$ with $h \notin G$. Let $x$ be any element of $G$. Then $h \notin G \Rightarrow\langle h, x\rangle \notin R_{\Im} \Rightarrow\langle x, h\rangle \in R_{\Im} \Rightarrow x$ $\in H$, because $h \in H \in J$. Thus $G \subset H$ and $J$ is nested.

Using Lemma 1 together with Theorem A, we have the following

Corollary 1. A topological space $(X, J)$ is minimal $T_{0}$ iff $R_{\mathfrak{J}}$ is linear and $\{\{y: y<x\}: x \in X\} \cup\{X\}$ is a base for $J$ (where $a \leqq b$ iff $\left.\langle a, b\rangle \in R_{\mathfrak{3}}\right)$.

Proof. If $J$ is minimal $T_{0}$, then $J$ is $T_{0}$ and nested by Theorem A. Thus $R_{\mathfrak{J}}$ is linear by Lemma 1 , because a linear order is just a totally ordered partial order. For each $x \in X$,

$$
\begin{aligned}
\sim\{\bar{x}\} & =\sim\{y: \text { every neighborhood of } y \text { contains } x\} \\
& =\sim\{y: x \leqq y\} \\
& =\{y: y<x\}
\end{aligned}
$$

because $R_{\Im}$ is linear. Therefore, $\{\{y: y<x\}: x \in X\} \cup\{X\}$ is a base for 3 .

Conversely, if $R_{\mathfrak{T}}$ is linear and $\{\{y: y<x\}: x \in X\} \cup\{X\}$ forms a base for $J$, then $J$ is $T_{0}$ and nested by Lemma 1 . Furthermore, since $R_{\mathfrak{J}}$ is linear,

$$
\sim\{\bar{x}\}=\sim\{y: x \leqq y\}=\{y: y<x\}
$$

for each $x \in X$, and $\{\sim\{\bar{x}\}: x \in X\} \cup\{X\}$ is a base for $J$. Thus $J$ is minimal $T_{0}$. 
LeMma 2. Let $\varphi: J \rightarrow R_{\mathfrak{J}}$ be restricted to the minimal $T_{0}$-topologies on $X$. For each linear ordering, $R$, of $X$, let $\mu(R)$ be the topology with base $\left\{\left\{y: y<_{R} x\right\}: x \in X\right\} \cup\{X\}$. Then $\mu$ is an inverse for $\varphi$ and hence $\varphi$ is a 1-1 map of the minimal $T_{0}$ topologies onto the linear orders of $X$.

Proof. If $\Im$ is a minimal $T_{0}$-topology, then $R_{\mathfrak{J}}=\varphi(J)$ is linear. By Corollary 1 , a base for $J$ is $\left\{\left\{y: y<_{R_{y}} x\right\}: x \in X\right\} \cup\{X\}$. But by definition, this is also a base for $\mu\left(R_{\mathfrak{J}}\right)=\mu(\varphi(J))$. Therefore, $J=\mu(\varphi(J))$.

Now let $R$ be a linear order and let $J=\mu(R)$. Then $J$ has base $B=\left\{\left\{y: y<_{R} x\right\}: x \in X\right\} \cup\{X\}$. If $\langle a, b\rangle \in R$ and $N_{b}$ is any open neighborhood of $b$, then there is a set $B \in \mathbb{B}$ such that $b \in B \subset N_{b}$. If $B=X$, then clearly $a \in B \subset N_{b}$. If $B \neq X$, then $B=\left\{x: x<{ }_{R} c\right\}$ for some $c \in X$. Then $b \in B \Rightarrow a \leqq{ }_{R} b<_{R} c \Rightarrow a<{ }_{R} c \Rightarrow a \in B \subset N_{b}$. That is, $\langle a, b\rangle \in R_{\mathfrak{\jmath}}=\varphi(\mathfrak{J})$, and thus $R \subset \varphi(\mathfrak{J})$. Let $\langle a, b\rangle \in \varphi(\mathfrak{J})=R_{\mathfrak{J}}$. If $b<_{R} a$, then $\left\{x: x<{ }_{R} a\right\}$ is an open set in $J$ containing $b$ but not $a$, which contradicts $\langle a, b\rangle \in R_{\mathcal{J}}$. Therefore, because $R$ is linear, $a \leqq_{R} b,\langle a, b\rangle \in R$, and so $\varphi(J) \subset R$. Hence $R=\varphi(J)=\varphi(\mu(R))$, and $\varphi$ and $\mu$ are inverses.

TheOREM 1. A topology $\Im$ on a set $X$ is an order topology iff $(X, \mathfrak{J})$ is a $T_{1}$-space and $J$ is the least upper bound of two minimal $T_{0}$-topologies.

Proof. If $I$ is the order topology on $X$ associated with the linear order $R$, then $J$ is generated by all sets of the forms

$$
\{y: y<x\} \text { and }\{y: x<y\} .
$$

It is clear from the linearity of $R$ that $J$ is $T_{1}$. Let $J_{1}$ be the topology with base

$$
B_{1}=\{\{y: y<x\}: x \in X\} \cup\{X\},
$$

and let $J_{2}$ be the topology with base

$$
\mathrm{B}_{2}=\{\{y: y>x\}: x \in X\} \cup\{X\} .
$$

Then, by Lemma $2, \Im_{1}$ and $\Im_{2}$ are minimal $T_{0}$-topologies, and $R_{J_{1}}=R$ while $R_{J_{2}}=R^{-1} . \otimes_{1} \cup B_{2}$ is a subbase for $J_{1} \vee J_{2}$, but is also a subbase for $J$. Therefore, $J=J_{1} \bigvee J_{2}$ is the least upper bound of two minimal $T_{0}$-topologies.

Conversely, suppose $J=J_{1} \vee J_{2}$ where $J_{1}$ and $J_{2}$ are minimal $T_{0^{-}}$ topologies and $\Im$ is $T_{1}$. Then $R_{\mathfrak{I}_{1}}$ and $R_{J_{2}}$ are linear. If $\langle a, b\rangle \in R_{\mathfrak{J}_{1}} \cap R_{\mathfrak{J}_{2}}$ where $a \neq b$, let $G$ be an open set in $J_{1} \bigvee J_{2}$ containing $b$. Then there exist sets $G_{1} \in J_{1}$ and $G_{2} \in J_{2}$ such that $b \in G_{1} \cap G_{2} \subset G$. But then $\langle a, b\rangle \in R_{\mathfrak{I}_{1}} \cap R_{\mathfrak{I}_{2}} \Rightarrow a \in G_{1}$ and $a \in G_{2} \Rightarrow a \in G_{1} \cap G_{2} \subset G$. That is, every neighborhood of $b$ in $J$ contains $a$, which is impossible when $J$ is $T_{\mathbf{1}}$. Therefore, for $a \neq b$, 


$$
\langle a, b\rangle \in R_{J_{2}} \Rightarrow\langle a, b\rangle \notin R_{J_{1}} \Rightarrow\langle b, a\rangle \in R_{J_{1}} \Leftrightarrow\langle a, b\rangle \in R_{{J_{1}}_{1}}^{-1}
$$

Hence $R_{J_{2}}=R_{\mathfrak{J}_{1}}^{-1}$, and, since $\Im_{1}$ and $J_{2}$ are minimal $T_{0}$, they have bases $B_{1}=\{\{y: y<x\}: x \in X\} \cup\{X\}$ and $B_{2}=\{\{y: y>x\}: x \in X\} \cup\{X\}$, respectively, where $a \leqq b$ iff $\langle a, b\rangle \in R_{J_{1}}$. Therefore, $\Theta_{1} \cup{\Theta_{2}}_{2}$ is a subbase for $J_{1} \vee J_{2}=J$. But $\Theta_{1} \cup \beta_{2}$ also generates the order topology on $\left(X, R_{\mathfrak{I}_{1}}\right)$, which must therefore be $\mathfrak{J}$.

3. Proof of Theorem 2. Our characterization of the reals hinges on two well-known ideas: first of all, on the correspondence between order and topological properties of ordered spaces, and secondly, on the characterization of the natural order on the reals developed by Cantor and Hausdorff. We adopt the following definitions of some common order properties:

Definition 2. Let $R$ be a linear order on $X$. Then

(i) $(X, R)$ is complete iff every subset of $X$ bounded above has a least upper bound;

(ii) $\mathrm{A} \subset X$ is dense in $(X, R)$ iff whenever $x<_{R} y$ there is an element $z \in A$ such that $x<{ }_{R} z<{ }_{R} y$;

(iii) $(X, R)$ is dense iff $X$ is dense in $(X, R)$.

The following relations between these order properties and the corresponding order topologies are well known:

THEOREM B. If $R$ is a linear order on $X$ and $J$ is the associated order topology, then $(X, J)$ is connected iff $(X, R)$ is complete and dense.

THEOREM C. If $R$ is a dense linear order on $X$ and $I$ is the associated order topology, then $(X, J)$ is separable iff $(X, R)$ has a countable dense subset.

Eilenberg in 1941 [2, p. 40] showed that if the order topology associated with $(X, R)$ is connected, then $(X, R)$ is dense and complete. The actual characterization (Theorem B) appears in Murty [8, p. 157]. Theorem C was verified in the proof of Eilenberg's Theorem $6.1[2$, p. 43$]$, although not stated as a separate theorem.

Hausdorff in 1914 [6, p. 101] generalized Cantor's characterization $[1$, p. 511$]$ of the order on the closed interval $[0,1]$ to obtain the following characterization of the natural order on the reals:

THEOREM D. An ordered set $(X, R)$ is order isomorphic to the reals 
with the usual ordering iff $(X, R)$ is a complete linear order with no greatest or least element and $X$ has a countable subset dense in $(X, R)$.

Theorem 2. A topological space $(X, \Im)$ is homeomorphic to the reals with the usual topology iff $(X, J)$ is a connected, separable, $T_{1}$-space, and $J$ is the least upper bound of two noncompact minimal $T_{0}$-topologies.

Proof. Let $(R, \mathcal{u})$ be the reals with the usual topology and let $R$ be the usual order on $R$.

If $(X, \mathfrak{J})$ is homeomorphic to $(R, \mathcal{U})$, we may assume that $(X, \mathfrak{J})$ $=(R, \mathcal{U})$. Certainly, $(R, \mathcal{U})$ is connected, separable, and $T_{1}$. By Theorem 1 , since $U$ is the order topology associated with $R, \mathcal{U}$ $=J_{1} \bigvee J_{2}$ where $J_{1}$ and $J_{2}$ are minimal $T_{0}$-topologies. Furthermore, by the construction of $J_{1}$ and $J_{2}$ in the proof of Theorem 1 , it is clear that neither $J_{1}$ nor $J_{2}$ is compact.

Conversely, suppose that $(X, \Im)$ is a connected, separable, $T_{1}$-space and that $J$ is the least upper bound of two noncompact minimal $T_{0^{-}}$ topologies, $J_{1}$ and $J_{2}$. Let $S=R_{J_{1}}$ be the order associated with $J_{1}$. Then $S$ is linear and $R_{J_{2}}=S^{-1}$ by the proof of Theorem 1 . Also by Theorem $1, J$ is the order topology on $X$ associated with $S$. By Theorem $\mathrm{B}$, since $(X, J)$ is connected, $(X, S)$ is complete and dense. Then, since $(X, J)$ is also separable, $(X, S)$ has a countable dense subset by Theorem $C$. If $(X, S)$ had a greatest element $b$ and $a$ were any open cover of $X$, then we would have $b \in A_{b}$ for some $A_{b} \in Q$. But then $\langle x, b\rangle \in S$ for all $x \in X \Rightarrow x \in A_{b}$ for all $x \in X$, by definition of $S$. Thus $A_{b}=X$ and $\left\{A_{b}\right\}$ would be a finite subcover, which is a contradiction. Therefore, $(X, S)$ has no greatest element. In like manner, $\left(X, S^{-1}\right)$ has no greatest element. That is, $(X, S)$ has no greatest and no least element. In summary, $(X, S)$ is a complete linear order with no greatest or least element and $X$ has a countable subset dense in $(X, S)$. Thus $(X, S)$ is order isomorphic to $(R, R)$ by Theorem $\mathrm{D}$. But since $(X, \mathfrak{J})$ and $(R, \mathcal{U})$ are just the order topologies on $X$ and $R$ associated with $S$ and $R$, respectively, it is clear that $(X, \mathfrak{J})$ is homeomorphic to $(\boldsymbol{R}, \mathcal{U})$.

\section{REFERENCES}

1. Georg Cantor, Beiträge zur Begründung der transfiniten Mengenlehre, Math. Ann. 46 (1895), 481-512.

2. Samuel Eilenberg, Ordered topological spaces, Amer. J. Math. 63 (1941), 39-45. MR 2, 179.

3. S. P. Franklin and G. V. Krishnarao, On the topological characterization of the real line, Department of Mathematics, Carnegie-Mellon University, Report \#69-36, 1969. 
4. Orrin Frink, Topology in lattices, Trans. Amer. Math. Soc. 51 (1942), 569-582. MR 3, 313.

5. Alfred Haar and Dénes König, Über einfach geordnete Mengen, J. Reine Angew. Math. 139 (1911), 16-28.

6. Felix Hausdorff, Grundzüge der Mengenlehre, Veit, Leipzig, 1914; reprint, Chelsea, New York, 1949; 2nd ed., Berlin, 1927. MR 11, 88.

7. Roland E. Larson, Minimal $T_{0^{-}}$paces and minimal $T_{D^{-}}$paces, Pacific J. Math. 31 (1969), 451-458.

8. A. S. N. Murty, Simply ordered spaces, J. Indian Math. Soc. 13 (1949), 152158. MR 11, 532.

9. Oystein Ore, Some studies on closure relations, Duke Math. J. 10 (1943), 761785. MR 5, 170.

10. Ki-Hyun Pahk, Note on the characterizations of minimal $T_{0}$ and $T_{D}$ spaces, Kyungpook Math. J. 8 (1968), 5-10. MR 38 \#2726.

11. M. Christian Pauc, Directions, contingent et paratingent dans les espaces distanciés, C. R. Acad. Sci. Paris 203 (1936), 153-155.

12. W. Sierpiński, L'arc simple comme un ensemble de points dans l'espace d $m$ dimensions, Ann. Mat. Pura Appl. (3) 26 (1917), 131-150.

13. A. K. Steiner, The lattice of topologies: Structure and complementation, Trans. Amer. Math. Soc. 122 (1966), 379-398. MR 32 \#8303.

14. W. J. Thron, Topological structures, Holt, Rinehart and Winston, New York, 1966. MR 34 \#778.

15. A. J. Ward, The topological characterization of an open linear interval, Proc. London Math. Soc. (2) 41 (1936), 191-198.

University of Colorado, Boulder, Colorado 80302 